\title{
Epidemiology and Outcome of Acute Bacterial Meningitis Among Children in Saudi Haospital Hajjah, Northwest Territories of Yemen
}

\author{
Ahmed Hamood Alshehari, Abdulhakim Ali Mohammed Al-Selwi*, \\ Mohamed Abdulhadi Albahloly \\ Department of Pediatrics, Faculty of Medicine, Dhamar University, Dhamar, Yemen \\ Email address: \\ shehari250@yahoo.com (A. H. Alshehari), hakimselwi@gmail.com (A. A. M. Al-Selwi), moham14@live.com (M. A. Albahloly) \\ *Corresponding author
}

\section{To cite this article:}

Ahmed Hamood Alshehari, Abdulhakim Ali Mohammed Al-Selwi, Mohamed Abdulhadi Albahloly. Epidemiology and Outcome of Acute Bacterial Meningitis Among Children in Saudi Haospital Hajjah, Northwest Territories of Yemen. American Journal of Pediatrics. Vol. 4, No. 3, 2018, pp. 56-60. doi: 10.11648/j.ajp.20180403.13

Received: May 16, 2018; Accepted: July 3, 2018; Published: July 30, 2018

\begin{abstract}
This is a retrospective descriptive study carried out in Saudi Hospital Hajjah between July 2016 and December 2017: The aim of this study is to assess the etiology and outcome of acute bacterial meningitis among children in Saudi Hospital Hajjah. We included in this study all children aged one month or more to 12 years who had symptoms and signs suggested acute bacterial meningitis confirmed by lumber puncture of cerebrospinal fluid for cells, biochemistry and culture. The data of all patients were retrieved from the hospital records and patient's charts. These data included age, clinical presentation, etiologic microorganism and outcome. A total of 196 patients who had confirmed bacterial meningitis was recorded. Of these, 71 patients $(36.2 \%)$ were aged under 12 months, $25 \%$ aged between $1-2$ years old, $17.9 \%$ between $3-5$ years and $20.9 \%>5$ years. Cerebrospinal fluid culture was positive among $94.9 \%$ of patients. Among the positive isolates, $43.5 \%$ were having Nesseria meningitidis, $34.9 \%$ Streptococcus pneumonia, $18.2 \%$ Hemophillus influenza type b, 2.7\% group $B$ Streptococci and $0.5 \%$ E.coli. There were 19 patients died giving the case fatality rate as $9.7 \%$. Of those, $47.3 \%$ had aged less than 12 months, 31.5\% aged $1-2$ years, one child (5.2\%) aged 4 years and 3 cases $(15.8 \%)$ were $>5$ years. Eleven cases of deaths $(57.9 \%)$ had s. pneumonia infections. There were 24 patients $(12.2 \%)$ of survivors developed neurological complications. This study demonstrates that there is no change in the epidemiology of the main causes of acute bacterial meningitis among children compared to that reported prior to introduction Hib and pneumococcal vaccines indicating a limited coverage of vaccination in the Northwest Territories of Yemen. The case fatality rate noted in this study is relatively low, but aggressive efforts by the health care system both to vaccinate and to early treat infected children, meningitis incidence and mortality will decline.
\end{abstract}

Keywords: Acute Bacterial Meningitis, Vaccine, Yemen

\section{Introduction}

Acute bacterial meningitis $(A B M)$ is a severe infectious disease of the membranes lining the brain and spinal cord. It is an important cause of childhood mortality and those who survive are at a higher risk of developing permanent neurological disability [1]. There are three main causative organisms accounting for over $90 \%$ of the world cases of ABM. These are Nesseria meningitidis, Streptococcus pneumonia and Hemophilus influenza type b (Hib) [2].
The clinical presentation of $\mathrm{ABM}$ is usually different according to the age of patients, the causative bacteria and the duration of symptoms [2]. Neonates often present with nonspecific symptoms such as irritability, poor feeding, respiratory distress, pale or marble skin and hyper- or hypotonia [3, 4]. Children beyond neonatal age, ABM typically begins with fever, chills, vomiting, photophobia and severe headache [5]. Because of absence of the classical 
signs and symptoms, the diagnosis of $\mathrm{ABM}$ in children is challenging and the definitive diagnosis is cerebrospinal fluid (CSF) analysis and culture [6]. There are also several tests that can be performed in suspected ABM cases such as blood culture, a throat swab, polymerase chain reaction (PCR) among others [6].

Treatment of ABM is dependent entirely on the antimicrobial therapy specific for the infecting microorganism. Despite the presence of vaccine preventable causes of this disease, the incidence of ABM and associated deaths in resource-limited countries continues to exceed those for developed countries [7]. Worldwide, the epidemiology of $A B M$ is changing rapidly due to introduction of an effective vaccination. Hib has now been virtually eradicated as a cause of childhood meningitis in countries where Hib vaccine is routinely given during infant's immunization schedule [8]. Similarly, there has been significant change of the disease profile after introduction of pneumococcal vaccine [8, 9]. Not only are vaccination against those microorganisms safe and cost-effective but it also prevents unnecessary death and disability. Yemen is one of the least developing countries and has a very low-income compared with the neighboring countries. Hib vaccine has been introduced in the country 2005 and pneumococcal vaccine (PCV) was introduced $n$ 2011[10]. The objective of this study is to assess the etiology and outcome of ABM among children aged beyond a month to 12 years in Saudi Hospital Hajjah.

\section{Methods and Materials}

A retrospective analysis of all children admitted to Saudi Hospital Hajjah with the diagnosis of ABM over 18 months period from July 2016 to December 2017 was carried out.

\subsection{Study Setting}

Saudi Hospital Hajjah (SHH) is located in Hajjah governorate about $200 \mathrm{~km}$ Northwest to Sana 'a capital. The hospital is provided by the kingdom of Saudi Arabia to help Yemenis patients in those rural regions, completely financed, and supervised by the Saudi government. It is an emergency hospital works over 24 hours and serves all population of Hajjah, Hodeidah, S'adah and Amran governorates, in addition to the nearby villages of $\mathrm{Al}$ Mahweet governorate. It provides the medical service in all medical branches at no cost and contains 93-beds including 10-beds for pediatric ward and 15-beds for nursery in addition to emergency department with a capacity of 80 -beds. It is well equipped with a reliable laboratory, blood bank and radiological department. The working staff are mixed of Yemeni and external doctors, nurses and technicians. The hospital has an accurate recording system and follows a strict policy in admission and long term follow-up.

\subsection{Study Methods}

The pediatric charts of all children infected by ABM were reviewed and the data were recorded on a standard data collection form. The study was approved by the hospital ethics committee. For inclusion in this study, pediatric patients had to meet the following criteria: (a) age $\geq$ a month; (b) symptoms and signs suggestive of ABM; and (c) positive cerebrospinal fluid (CSF) culture or positive blood culture for an identified organism accompanied by abnormal CSF analysis. We excluded patients in whom the confirmed diagnosis of $\mathrm{ABM}$ was not established and those patients in whom viral meningitis is evidenced by normal or increased lymphocyte count, normal glucose, and total protein. Data collected included age, sex, weight of children, symptoms and signs, causative microorganisms, antibiotic therapy, complications at the time of discharge and final outcome. In this hospital, cerebrospinal fluid is used for culture and bacterial sensitivity and analysis for leukocyte count, glucose and total protein as standard. Gram stain is mostly performed for the suspected cases. The data collected were analyzed using SPSS 21.0, and tabulated using frequency distribution tables as proportions or percentages. Suitable statistical tests were applied as applicable. A $p$ value $\leq 0.05$ is considered statistically significant.

\subsection{Follow-up}

The policy of hospital adopt arrangement of a long-term regular follow-up for all patients after discharge depending on the condition and sequelae encountered. Follow-up visits consist of detailed history, physical and neurological examination and investigation as indicated.

\section{Results}

One hundred ninety-six cases of acute bacterial meningitis for children from May 2016 to December 2017 were recorded. The age of children ranged between $\geq$ a month up to 12 years old. The higher incidence of meningitis $(36.2 \%)$ was noted among children aged 1-12 months. Of all cases, $128(65.3 \%)$ were males and the male to female ratio is $(1.9: 1)$ (table 1).

Table 1. Distribution of pediatric patients with ABM according to age group.

\begin{tabular}{lll}
\hline Age group (years) & No. & \% \\
\hline Months - 12 months & 71 & 36.2 \\
$1-2$ & 49 & 25 \\
$3-5$ & 35 & 17.9 \\
$>5-12 *$ & 41 & 20.9 \\
Male $128(65.3 \%)$ & & \\
Total & 196 & 100 \\
\hline
\end{tabular}

${ }^{*} \mathrm{P}$ value of the incidence of ABM among children aged $\leq 5$ years $(<0.001)$ compared with those aged $>5$ years; $95 \%$ CI 72.62 to 84.48 .

The commonest clinical presentation was fever $(87.7 \%)$, lethargy/irritability (71.9\%) and vomiting (47.4\%). Table 2 shows the clinical presentation of pediatric patients. 
Table 2. Clinical presentation of pediatric patients presented with ABM.

\begin{tabular}{lll}
\hline Symptoms & No. & \% \\
\hline Fever & 172 & 87.7 \\
Lethargy / irritability & 141 & 71.9 \\
Vomiting & 93 & 47.4 \\
Neck stiffness & 57 & 29 \\
Seizure & 67 & 34.1 \\
Altered mental status & 20 & 10.2 \\
Others & 8 & 4 \\
Total & 196 & 100 \\
\hline
\end{tabular}

CSF culture was positive for 186 cases (94.9\%) and negative for 10 cases $(5.1 \%)$. Of the positive CSF growth, there were 81 patients $(43.5 \%)$ had Nesseria meningitidis, streptococcus pneumonia were present in 65 patients (34.9\%), Hemophilus influenza 34 patients (16.2\%), group B streptococcus 5 cases $(2.7 \%)$ and E. coli $(0.5 \%)$. Table 3.

Table 3. Distribution of the CSF culture results according to age.

\begin{tabular}{|c|c|c|c|c|c|}
\hline & $<1 \mathrm{yr}(\mathrm{n}=71)$ & $1-2$ yr $(n=49)$ & $3-5$ yr $(n=35)$ & $>5 \mathrm{yr}(\mathrm{n}=41)$ & Total (\%) \\
\hline S. pneumonia & 29 & 21 & 7 & 8 & $65(33.1)$ \\
\hline H. influenza & 21 & 8 & 5 & 0 & $34(17.3)$ \\
\hline N. meningitidis & 17 & 18 & 18 & 28 & $81(41.3)$ \\
\hline Group B strept. & 3 & 1 & 1 & 0 & $5(2.6)$ \\
\hline E. coli & 0 & 1 & 0 & 0 & $1(0.5)$ \\
\hline No isolated & 1 & 0 & 4 & 5 & $10(5.1)$ \\
\hline
\end{tabular}

Of the positive and negative CSF growth, $N$. meningitidis was the commonest causative agent of bacterial meningitis constituted (41.3\%) followed by S. pneumonia (33.1\%), and H. influenza (17.3\%). The incidence of ABM among children aged $\leq 5$ years was $79 \%$ which is considered statistically significant compared to incidence among age $>5$ years $(p$ $<.0 .001)$.

There were 19 pediatric patients death giving case fatality rate as $9.7 \%$. Of those, 9 cases $(47.3 \%)$ were aged under 12 months, 6 cases $(31.5 \%)$ aged $1-2$ years old, one child was 4 years and 3 cases $(15.8 \%)$ were $>5$ years old. S. pneumonia was responsible for 11 cases of deaths $(57.9 \%), N$. meningitidis 4 cases, H. influenza 3 cases $(15.8 \%)$ and group $B$ streptococcus one case $(5.2 \%)$. Among those who survived, 24 children $(12.2 \%)$ developed neurological sequelae. Table 4.

Table 4. Distribution of children outcome according to age and bacterial pathogens.

\begin{tabular}{lllll}
\hline Variable & $<$ year & $\mathbf{1 - 2}$ & $\mathbf{3 - 5}$ & Total (\%) \\
\hline Deaths (total) & $(9)$ & $(6)$ & $(1)$ & $(3)$ \\
S. pneumonia* & 7 & 3 & 1 & 0 \\
N. meningitidis & 1 & 1 & 0 & 2 \\
H. influenza & 1 & 1 & 0 & 4 \\
Group B strept. & 0 & 1 & 0 & 1 \\
Neurological complication & 13 & 6 & 4 & 0 \\
\hline
\end{tabular}

S. pneumonia contributed significantly to death (95\% CI 1.46 to $33.74, p<0.001$ ).

\section{Discussion}

In this study, CSF culture was the diagnostic standard tool for ABM. The distribution of the epidemiologic pathogens among infected children studied showed that $41.3 \%$ had $N$. meningitidis, $33.1 \%$ had S. pneumonia, $17.3 \%$ had $H$ influenza $b$ and $2.6 \%$ had group $B$ streptococcus. Al Khorasani A and colleagues in old study conducted in the Northern rural area of Yemen (Sa'dah governorate) reported that in 160 pediatric patients who had confirmed ABM, $52.9 \%$ had $N$. meningitidis, $30.1 \%$ S. pneumonia and $15 \%$ had $H$. influenza [11], similar to the results of this study. Recent hospital-based study from neighboring region, Taif, Saudi Arabia found comparable frequency of pathogens causing ABM [12].

The result of this study shows that among children aged 1-
5 years who had a positive CSF culture; S. pneumonia was the commonest pathogen isolated constituted $36.7 \%$ followed by $N$. meningitidis $34.1 \%$, H. influenza $21.9 \%$ and group B streptococcus $3.2 \%$. These findings are similar to $\mathrm{Al}$ Khorasani A study [11]. S. pneumonia, and meningococcal infections are the most frequent meningitis-causing pathogens among children aged $\geq$ months up to 5 years old [13-15]. Tegene B from Ethiopia reported the predominant of S. pneumonia accounting for $43.3 \%$ of the pathogens causing $\mathrm{ABM}$ in pediatric [16]. In a study conducted in Cameron, it is found that $H$. influenza was the most incriminated pathogen of ABM among children of age $>2$ months to 2 years (39.2\%), followed by S. pneumonia $31.6 \%$, and $N$. meningitidis $10.5 \%$. The authors explained the high frequency of $H$. influenza instead of the predominance of $S$. pneumonia as a consequence to selection bias because they have included only the patients who had a positive culture or 
positive soluble antigen and also to the lack of Hib vaccination in most patients [17]. However, in the literature, $S$. pneumonia is documented to be the most frequent isolate microorganism causing ABM [18].

In Yemen, $H$. influenza type $b$ (Hib) vaccine has been introduced in 2005 and the pneumococcal vaccine introduced in 2011[11]. These vaccines target the most common bacterial causes of meningitis, S. pneumonia and $H$. influenza. A reduction of Hib meningitis post vaccination reported as $75 \%$ in Morocco [19, 9]. Hib vaccine has been shown to be efficacious in more than $95 \%$ in diverse population around the world [9]. Banajeh SM et al in a study carried out in Sana a, compared childhood very severe pneumonia and meningitis pre and post Hib vaccine found a statistically significant reduction of meningitis $(34 \%, 95$ CI $12-49, p<0.02)$ [10].

Regarding the impact of the vaccination on the burden of disease, it is interesting that comparing the proportion of the main common causes of ABM (i.e. S. pneumonia and Hib) in this study with Al Khorasani findings [10] who conducted the study in Sa'dah governorate located within the geographical region of this study immediately (2006) before Hib vaccine introduction, it is observed that there is no measurable declining of these two invasive pathogens indicating that the vaccination coverage in these areas is inadequate and questionable.

We therefor suggest that additional coverage of both vaccines in all rural regions along country using mobile vaccination teams with media promotion is very important to be arranged promptly. In addition, there is a need to increase the fixed vaccination stations set up at the existing primary health centers.

Although CSF culture remains the gold standard diagnostic technique for bacterial meningitis [20], several studies have been reported a substantial percentage of culture negative $[21,18]$. The current study observed a failure to isolate in $5.1 \%$. This finding might be related to either partial antibiotic treatment pre hospitalization, or improper laboratory handling of the specimens, which emphasizes the significant need to improve the diagnostic capabilities.

CSF culture allows for determination of bacterial type and sensitivity to the most effective antimicrobial drug however, the gram stain smear is recommended as a quick method to identify the bacterial etiology [22]. ABM is a medical emergency so rapid and correct diagnosis is the crucial step in the management to avoid potential delay with the conventional culture method for at least 48 hours. It is better therefore to recommend a gram stain testing in our setting to allows early antibiotic administration. Male sex was predominant in this study supporting the findings of other studies [23, 24]. The explanation of this observation is unclear.

It is difficult to estimate the incidence of ABM among the population in these areas because $\mathrm{SHH}$ is a referral hospital, serves 4 provinces including Hajjah, Sa dah, Hudeidah and Amran with a population of approximately 5209909. However, because the hospital exclusively provides services at no cost including investigations and medications this could attract only those patients who have the ability to cover the travel costs, resulting in uneven number of patients seeking hospital care. The situation is further complicated by the fact that many patients may die at home without recognition. In addition, a high number of suspected cases of meningitis are referred to local hospitals or primary health centers that have fewer resources and unreliable laboratory testing thus, the diagnosis may be missed.

The present study demonstrates that $\mathrm{S}$ pneumonia was the most common bacteria causing death contributed to $57.9 \%$ cases, followed by $H$. influenza $21 \%$ and $N$. meningitidis $15.8 \%$. The overall case fatality rate (CFR) is $9.7 \%$, similar to another study $[25,11]$. CFR observed in this study is lower than $31.29 \%$ reported in a study carried out in India [26] and $18.5 \%$ in Cameron [17]. However, there are various factors affecting the outcomes such as age, severity of the disease, virulence of the infecting microorganisms, time elapsed since the onset of disease till a specific antibiotic administration and quality of care [26]. In the present study the majority of deaths occurred for children under a year, and thus the risk factors of death noticed in this study are: younger age $<12$ months, delay in starting medication $>3$ days since the onset of disease, presence of $\mathrm{S}$ pneumonia causing meningitis and severe degree of symptoms at time of hospitalization.

The antibiotic selection and course followed in this study was based on the hospital protocol, which depends on up to date scientific evidence for the best medical practice released by literature. The adjunctive dexamethasone prophylaxis is given for selected patients but we could not examine its beneficial on the outcome because of the retrospective nature of this study.

The main limitation of this study is its retrospective design with lack of information on antibiotic use prior to hospital admission. In addition, there is no information regarding bacterial resistance to antibiotic administered. However, this could be explained by the fact that all patients ultimately received antibiotics according to the results of CSF susceptibility pattern.

\section{Conclusion}

This study demonstrates that the relative frequencies of the main causes of acute bacterial meningitis among children still having the same pattern as reported prior to introduction of Hib and pneumococcal vaccines reflecting unremarkable success of the vaccination program in these Northwest part of Yemen.

While CSF culture yield a high number of bacterial growth, a few cases of culture negative have been observed in this study, suggesting that additional CSF analysis such as gram stain, and antigen latex agglutination tests are required in setting where the facility for polymerase chain reaction is unfeasible. The case fatality rate noted in this study is relatively low, but however aggressive eforts by the health care system both to vaccinate and to early treat infected children, meningitis incidence and mortality will decline.

\section{Conflicts of Interest}

The authors declare that they have no conflicts of interest. 


\section{References}

[1] Jarousha AMA, Afifi AA. Epidemiology and risk factors associated with developing bacterial meningitis among children in Gaza Strip. Iran J Public Health 2014; 43: 11761183.

[2] Erum A, Zafar MZ, Rasool S, Ali Z, Yousaf K, et al. Different Incidences of Acute Bacterial Meningitis in Children of Central Punjab in Pakistan. J Neurol Neurosci. 2017; Vol. 8 No. S4:231

[3] Okike IO, Johnson AP, Henderson KL, Blackburn RM, Muller-Pebody B, Ladhani SN, et al. Incidence, etiology, and outcome of bacterial meningitis in infants aged $<90$ days in the United kingdom and Republic of Ireland: prospective, enhanced, national populationbased surveillance. Clin Infect Dis 2014;59:e150-7.

[4] Gaschignard J, Levy C, Romain O, Cohen R, Bingen E, Aujard Y, et al. Neonatal bacterial meningitis: 444 cases in 7 years. Pediatr Infect Dis J 2011; 30: 212-7.

[5] Snaebjarnardottir K, Erlendsdottir H, Reynisson IK, Kristinsson K, Halldórsdóttir S, Hardardóttir $\mathrm{H}$, et al. Bacterial meningitis in childrenin Iceland, 1975-2010: a nationwide epidemiological study. Scand J Infect Dis 2013; 45: 819-24.

[6] El Bashir H, Laundy M, Booy R. Diagnosis and treatment of bacterial meningitis. Downloaded from http://adc.bmj.com/ on March 9, 2018 - Published by group.bmj.com

[7] Li Y, Yin Z, Shao Z, Li M, Liang X, Sandhu HS, et al. Population-based surveillance for bacterial meningitis in China, September 2006-December 2009. Emerg Infect Dis 2014; 20(1):61-9. pmid: 24377388

[8] Agrawal S, Nadel S. Acute bacterial meningitis in infants and children: epidemiology and management. Paediatr Drugs. 2011; 13: 385-400.

[9] Ramesh Verma, Pardeep Khanna, Suraj Chawla, Mohan Bairwa, Shankar Prinja \& Meena Rajput. Hemophilus influenzae type b (Hib) vaccine: An effective control strategy in India, Human Vaccines 2011; 7:11, 1158-1160, DOI: 10. 4161/hv. 7. 11. 17683.

[10] Banajeh SM, Ashoor O and Al-Magramy AS: Childhood very severe pneumonia and meningitis-related hospitalization and death in Yemen, before and after introduction of $\mathrm{H}$. influenzae type b (Hib) vaccine. East Mediterr Health J. 20:431-441. 2014.

[11] Al Khorasani A, Banajeh S. Bacterial profile and clinical outcome of childhood meningitis in rural Yemen: a 2-year hospital-based study. J Infect. 2006; 53: 228-234.

[12] Nour M, Alaidarous A. Simultaneous Detection of Bacterial Meningitis in Suspected. Arch Clin Microbiol. 2017, 8:3.

[13] Kim KS. Acute bacterial meningitis in infants and children. Lancet 2010; 10: 32-42.

[14] Pick AM, Sweet DC, Begley KJ. A Review of Pediatric Bacterial Meningitis. US Pharmacist. 2016; 41: 41-45.

[15] Houri H, Pormohammad A, Riahi SM, asiri M J, Fatemeh Fallah F, DabiriH, Pouriran R. Acute bacterial meningitis in Iran: Systematic review and meta-analysis PLoS ONE 12(2): e0169617. doi: 10. 1371/journal. pone. 0169617.

[16] TegeneB, GebreselassieS, FikrieN. Bacterial Meningitis: a five-year retrospective study among patients who had attended at University of Gondar Teaching Hospital, Northwest Ethiopia. Biomedical Research and Therapy 2015, 2(5): 270278.

[17] Nguefack, S., Chiabi, A., Enoh, J., Djouberou, E. H., Mah, E., Kamga, K. K., Tatah, S. and Mbonda, E. Etiologies and Outcome of Children with Purulent Meningitis at the Yaounde Gyneco-Obstetric and Pediatric Hospital (Cameroon). Open Journal of Pediatrics, 2014; 4, 269-275.

[18] Khater WS, Elabd SH. Identification of Common Bacterial Pathogens Causing Meningitis in Culture Negative Cerebrospinal Fluid Samples Using RealTime Polymerase Chain Reaction. Int J Microbiol 2016; 2016: 4197187.

[19] Braikat M, Barkia A, El Mdaghri N, Rainey JJ, Cohen AL, Teleb N. Vaccination with Haemophilus influenzae type b conjugate vaccine reduces bacterial meningitis in Morocco. Vaccine. 2012 Mar 28; 30 (15):2594-9. PMID: 22306854.

[20] Wu HM, Cordeiro SM, Harcourt BH, et al. "Accuracy of realtime PCR, Gram stain and culture for Streptococcus pneumoniae, Neisseriameningitidis and Haemophilus influenza meningitis diagnosis," BMC Infectious Diseases, vol. 13, no. 1, article 26, 2013.

[21] van de Beek D., Cabellos C., Dzupova O., et al. ESCMID guideline: diagnosis and treatment of acute bacterial meningitis Clin. Microbiol. Infect. 22 (Suppl. 3): S37-S62, 2016.

[22] Al Bekairy AM, Al Harbi S, Alkatheri AM, Al Dekhail S, Al Swaidan L, Khalidi N. Bacterial meningitis: an update review. Afr J Pharm Pharmacol. 2014; 8(18): 469-478.

[23] Khan FH, Abu-Khattab M, Almaslamani EA, Hassan AA, Mohamed SF, Abdurrahman Ali Elbuzdi AAet al. Acute Bacterial Meningitis in Qatar: A Hospital-Based Study from 2009 to 2013. BioMed Research International 2017; 2017: 18.

[24] Fouad R, Khairy M, Fathalah W, et al. Role of clinical presentations and routine CSF analysis in the rapid diagnosis of acute bacterial meningitis in cases of negative gram stained smears. J Trop Med 2014; 2014: 213762.

[25] Mohanty N, Biswas T K, Satapathy S, Meher S k, Patro D. Etioclinical profile and outcome of acute bacterial meningitis in post neo natal U-5 children: a study from tertiary care center of coastal Odisha, India. Int J Res Med Sci. 2017 Jun; $5(6): 2519-2523$.

[26] Joardar S, Joardar GK, Mandal PK, Siddhartha Mani S. Meningitis in children: a study in medical college \& hospital, Kolkata. Bangladesh J Child Health 2012; Vol 36 (1): 20-25. 